Original Research Paper

\title{
Secondary User Sensing Time Optimization in Sensing- Transmission Scheduling for Dynamic Spectrum Access in Cognitive Radio
}

\author{
Inderdeep Kaur Aulakh and Renu Vig \\ University Institute of Engineering and Technology, Panjab University, Chandigarh, India
}

\author{
Article history \\ Received: 13-02-2014 \\ Revised: $13-07-2015$ \\ Accepted: 6-10-2015 \\ Corresponding Author: \\ Inderdeep Kaur Aulakh \\ University Institute of \\ Engineering and Technology, \\ Panjab University, Chandigarh, \\ India \\ Email: iaulakh $@$ rediffmail.com
}

\begin{abstract}
The constraint of limited frequency spectrum for wireless communication is very daunting. The radio spectrum licensed to permanent users is wasted at times when not used continuously. Cognitive radio technology involves a change in regulation of radio spectrum. In cognitive radio networks a secondary user network is designed for efficiently using gaps in spectrum and not causing at the same time harmful interference to license holding primary users. Opportunistic spectrum access involves the sensing of white spaces in the transmissions of Primary Users and their utilization by Secondary Users. There is a continuous tradeoff between the opposing goals of Primary User transmission protection and Secondary User throughput maximization. For a Secondary User, sensing is important so as to avoid collisions, hence packet loss. At the same time being too cautious and having long sensing periods can be costly. This paper presents optimization of Secondary User sensing time in sensing-transmission scheduling for dynamically accessing the spectrum in cognitive radios. The need to maximize the Secondary User access is achieved by optimizing its sensing time with respect to collision cost in a threshold based sensingtransmission structure using simulations. The results are presented in the form of traces of thresholds for different sensing times and collision costs. This scheme optimizes Secondary User throughput while safeguarding Primary User interests. This defines a standard to give an insight into the design of sensing-transmission scheduling in cognitive radio networks.
\end{abstract}

Keywords: Cognitive Radio, Threshold, Secondary User Sensing Time, Collision Cost, Sensing-Transmission Scheduling

\section{Introduction}

Today, spectrum is like real estate. It is a commodity which is fixed but the demand for it is increasing exponentially each day. Access to radio spectrum is difficult as it is controlled by a radio regulatory regime. Large parts of the radio spectrum are allocated to licensed radio services in a commandand-control way. Open access to most of the radio spectrum is not permitted. Only some small fractions of the radio spectrum, the unlicensed frequency bands, are openly available. The demand for additional spectrum is growing faster than the speed at which technology is able to increase spectrum efficiency and capacity in radio communications. Cognitive radio is not only a new radio technology; it also includes a revolutionary change in how the radio spectrum is regulated. Cognitive radios are designed for efficiently using shared spectrum and not causing at the same time harmful interference to incumbent (primary, license holding) radio systems (Fette, 2009; Berlemann and Mangold, 2009).

Secondary users have to cognitively use the gaps in spectrum, which keep on changing dynamically. In this technology the protection of primary user's transmission is very important. PU is not expected to change its access policy or co-operate with SU. Hence the Listen Before Talk (LBT) policy has been studied extensively 
by researchers. The SU listening is known as sensing and SU talk is called transmission. Though SU transmission is the only process which has to be maximized, it cannot be done without constraints. These are PU collisions and SU sensing durations because both these scenarios have to be restricted within limits.

Periodic sensing has been assumed in the IEEE 802.22, with both fast sensing and fine sensing. Prior works have considered per packet sensing, so the SU senses the PU channel at the beginning of each packet's transmission (Lai et al., 2007; Zhao et al., 2007; Liang et al., 2008; Wang et al., 2007). This type of sensing simplifies the design of the Physical and MAC layer but does not optimize the sensingtransmission scheduling. In many practical scenarios sensing is time consuming, for instance in IEEE 802.22 it is $10 \mathrm{~ms}$ for cordless microphone signals (Chen et al., 2008). This duration is $25 \mathrm{~ms}$ for field-sync detection of a standard Digital TV system (Cordeiro et al., 2006). This sensing time is not negligible compared to typical millisecond duration of a packet. Sensing overhead has to be tolerated because transmission without sensing would cause more collisions with PU. Thus in this study both benefit and penalty has been considered when designing sensing-transmissions schemes of SUs.

The effect of feedback from SU receiver has not been well studied as well. The SU transmitter cannot sense PU signals in the licensed band while transmitting, but the data received by the SU receiver can be affected by this PU interference. The SU receiver can be made to send an acknowledgement indicating successful reception of a packet when PU is not active. Similarly SU receiver can indicate failure of packet reception by sending a negative acknowledgement. This type of feedback can be used for taking better decisions by SU transmitters.

\section{Related Works}

The earlier models (Lai et al., 2007; Zhao et al., 2007; Liang et al., 2008; Wang et al., 2007) in this field have considered only periodic or per-packet sensing, which means that the SU senses the PU channel once before each packet is transmitted.

Liang et al. (2008; Wang et al., 2007), the authors have derived an optimal tradeoff between throughput and the false-alarm probability of a SU, by choosing a suitable sensing time. This work also assumes periodic sensing. In comparison, we have achieved a balance between minimizing the PU interruption and maximizing the SU transmission time based on collision cost, sensing result and feedback. Also general sensing-transmission structure has been considered in this study.

Kim and Shin (2008), the authors have proposed a scheme that optimizes both the probing period and the sensing sequence to maximize the detection of spectrum white spaces in multiple channels. They have assumed MAC-layer sensing scheduling for general PU idle/busy time distributions and not emphasized the interdependence of PU protection and SU performance which is integral to our research.

Chang and Liu (2007), the authors showed thresholdbased structure of the optimal strategy for channel guessing, probing and transmission. Temporal independence of channel states in a slotted structure has been assumed by the authors and during each slot, the state of the channel remains unchanged. In comparison, the aim of our paper is to compute the optimal sensing time in one PU channel. Also general un-slotted PU idle time distributions have been assumed

Chen et al. (2008), the authors assumed slotted PU activities and limited PU collision probability. They proposed an optimal operating point for the spectrum sensor, its access decision and channel selection. The whole observation history was utilized to find the optimal spectrum access policy. Thus the joint optimization problem was separated and reduced to an unconstrained problem.

The authors in (Zhao and Liu, 2008) extended the results in (Chen et al., 2008) to un-slotted PU activities. Zhao and Liu (2008) the authors assumed a per-packet sensing/transmission structure and the independence of PU state transition probability on time.

Huang et al. (2008) an optimal threshold-based policy has been derived for the exponential idle time distribution depending on its memory-less property by Huang et al. (2008). The approach cannot be applied to other idle time distributions because the PU state transition is dependent on time. Also, perfect sensing is critical in their study, but not in this study.

The models of (Huang et al., 2009a; 2009b) have not considered the effect of variation in cost of collision and the length of SU sensing time on optimal threshold for sensing-transmission structure. These factors are the primary constraints in this study.

Mokhtar et al. (2009) non perfect reporting channel has been considered in co-operative spectrum sensing. They have reduced the reporting errors in sensing, but imperfect feedback from SU receiver has not been considered to determine the sensing transmission scheduling.

The authors (Zhang and Tsang, 2011) have considered reward-based scheduling schemes and their optimality, but it's not clear how these algorithms can be applied in practice and how the actual sensing performances are.

Liu et al. (2012) have considered periodic sensing that too sequential of an in-band channel. Their focus has been mainly on deducing the optimal sensing frequency during an SU's active transmission on a PU licensed channel. Where-as in our paper we have considered feedback from 
$\mathrm{SU}$ receiver to check for $\mathrm{PU}$ transmission and other interferences during SU transmission.

In our earlier paper (Aulakh and Vig, 2014a) we have considered the effect of variation in reward of successful transmission and cost of collision on optimal threshold for sensing-transmission structure. The effect of Probability of false alarm and cost of collision has been considered in (Aulakh and Vig, 2014b). The effect of length of SU sensing time and cost of collision on optimal threshold for sensing-transmission structure has not been fine tuned. These are the primary constraints in this study.

\section{Discussion on Related Works}

In comparison with the earlier works, this paper is different in the following issues: Sensing-transmission structure of Secondary User is considered to be general with or without periodic/per packet sensing. PU idle time distribution has been assumed to be general and unslotted. The interdependence of PU protection and SU performance has been considered. Many of the earlier works have assumed perfect sensing. Imperfect sensing has been considered in this study. Also the SU receiver feedback in the form of acknowledgement/ negative acknowledgement has been considered. Effect of collision cost and SU sensing time on the optimal threshold has been fine tuned under all the above constraints.

\section{Methodology}

Firstly a system model is developed based on the constraints. Then it is simulated using MATLAB and the traces are compared with earlier works and then analyzed for new implications.

In order to develop a system model for SU sensing and transmission, based on semi-markov renewal theory, PU idle and busy period distributions are assumed to be general with means specified. Then based on probability theory and considering parameters like probability of false alarm, probability of correct detection of PU being busy, SU sensing period, SU packet length, probability of negative acknowledgement due to PU collision and probability of negative acknowledgement due to channel noise and interference the expressions for instantaneous benefit for successful SU packet transmission and penalty for collision with PU are deduced. The instantaneous utility obtained by SU at any time both for sensing and transmission is found next. Threshold is then defined as that probability of finding PU idle at which transmission utility exceeds sensing utility. This threshold is found to be dependent on various parameters including the SU sensing period.

To analyze the effect of SU sensing period the model is simulated in MATLAB and the threshold traces are obtained for different collision costs and SU sensing periods. Analyses of these results lead to determining the exact sensing-transmission scheduling for a given application.

\section{Development of System Model}

Assume a Secondary User transceiver pair is opportunistically accessing a PU channel. PU is unaware of SU activities hence transmits following an idle/busy pattern based on the semi-markov renewal theory. Thus its idle $(I)$ and busy $(B)$ timeperiods are independent, having distributions $f_{I}\left(\right.$.) and $f_{B}($.) with means $\bar{i}$ and $\bar{b}$.

The two activities of a SU transceiver are - sensing and transmitting. SU uses a spectrum sensor of energy or feature to find if PU is busy or idle at any instant. In case of non ideal sensing, Let:

- Probability of false alarm i.e., finding PU not idle when it is idle be Probf and

- Probability of finding PU busy when it actually is busy be Probd

- Let SU time unit be $\delta$ and for simplicity assume: $\delta=1$. Then we assume that

- $\mathrm{SU}$ has a fixed sensing period of: Tsense $\times \delta$ $=$ Tsense also

- SU has a fixed transmitting packet length of: Tpacket $\times \delta=$ Tpacket

- Sensing and Transmitting times are very less as compared to PU average idle and busy times

- Benefit for successful SU packet transmission is: Reward x Tpacket per $\delta$

- Penalty for collision with PU is: Collcost per $\delta$ or 1 in this case.

Though the sensing period and packet length have been assumed to be fixed, the SU can sense and transmit multiple times in succession, thus enhancing SU utility as desired. The PU can regulate the Collcost to control the SU access aggressiveness because the Average Utility function is Benefit minus Collcost. In this way the desired balance between SU spectrum usage and PU collision protection can be achieved and spectrum sharing between PU and SU can also be implemented.

It has been assumed that SU is aware of PU idle time distribution. Extensive measurements (Willkomm et al., 2008; Geirhofer et al., 2006), using various methods (Azzalini, 1981; Kim and Shin, 2008) can estimate this idle time distribution function This assumption is like assuming that PU idle time has exponential distribution with knowledge of its parameter, which has been done in earlier works.

In order to incorporate the feature of feedback in SU communication, the presence of acknowledgement and negative acknowledgement from the SU Receiver has been assumed. An acknowledgement validates packet transmission and its reception without collision. A 
negative acknowledgement can be due to collision with PU or interference or fading within the SU channel. Probability of negative acknowledgement due to PU collision is defined as $a_{l}$ and Probability of negative acknowledgement due to any other reason is $a_{0}$. Always: $a_{1}>a_{0}$.

\section{Formulation of Problem}

In order to efficiently utilize the white spaces in PU spectrum, sensing and transmission has to be scheduled by a SU transceiver as in Fig. 1. The SU can either sense multiple times or transmit multiple packets at any instant. This is a partially observable decision process as the SU does not have complete knowledge about PU being idle or busy.

\section{PU State Transition}

The Primary User state transition is not dependent on Secondary User actions but depends on time instant. If $t$ time has passed since PU's last transition from busy state to idle state and if it is assumed that the PU is still idle at time $t$, then probabilities that it will continue to remain idle are:

When SU is sensing:

$$
\operatorname{Prob}_{t}^{\text {Sense }}=\frac{1-F_{I}(t+T \text { sense })}{1-F_{I}(t)}
$$

When SU is transmitting:

$$
\operatorname{Prob}_{t}^{\text {Transmit }}=\frac{1-F_{I}(t+\text { Tpacket })}{1-F_{I}(t)}
$$

where, $F_{I}($.$) is cumulative distribution function of PU$ idle time.

The two probabilities depend on the time elapsed, which is $t+$ Tsense when sensing and $t+$ Tpacket when transmitting.

\section{Outcome State}

The SU has two actions: Sense and Transmit. The outcome of SU sensing can either be that PU is idle or it is busy. The outcome of SU transmission can be an acknowledgement or a negative acknowledgement.

Let conditional probability of PU being idle at time $\mathrm{t}$ be denoted by $\operatorname{Pr}_{t}$. Then the probability of PU remaining idle after sensing will be $P r_{t+T s e n s e}$ and probability of PU remaining idle after transmitting will be $P r_{t+T p a c k e t}$. These probabilities according to the Bayes' rule, will depend on the outcomes of each action and can be expressed as:

$$
\begin{aligned}
\operatorname{Pr}_{t+T \text { sense }}= & \frac{\operatorname{Pr}_{t} \times \operatorname{Prob}_{t}^{\text {Sense }} \times(1-\text { Probf })}{\operatorname{Pr}_{t} \times \operatorname{Prob}_{t}^{\text {Sense }} \times(1-\text { Probf })+} \\
& \left(1-\operatorname{Pr}_{t} \times \operatorname{Prob}_{t}^{\text {Sense }}\right)(1-\text { Probd })
\end{aligned}
$$

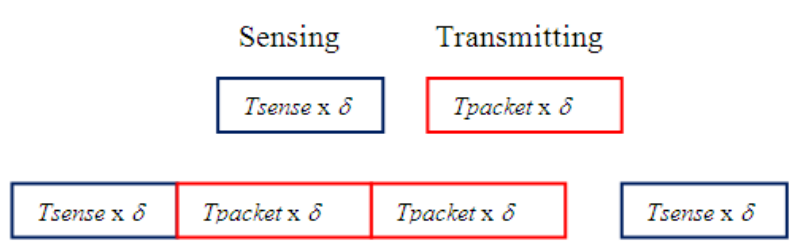

Fig. 1. Sensing-transmission scheduling

If outcome is PU idle:

$$
\begin{aligned}
P r_{t+T_{\text {Sense }}}= & \frac{\operatorname{Pr}_{t} \times \operatorname{Prob}_{t}^{\text {Sense }} \times \text { Probf }}{\operatorname{Pr}_{t} \times \operatorname{Prob}_{t}^{\text {Sense }} \times \text { Probf }+} \\
& \left(1-\text { Pr }_{t} \times \text { Prob }_{t}^{\text {Sense }}\right) \times \text { Probd }
\end{aligned}
$$

If outcome is PU busy:

$$
\begin{aligned}
\operatorname{Pr}_{t+\text { Tpacket }}= & \frac{\operatorname{Pr}_{t} \times \operatorname{Prob}_{t}^{\text {Transmit }} \times\left(1-a_{0}\right)}{\operatorname{Pr}_{t} \times \operatorname{Prob}_{t}^{\text {Transmit }} \times\left(1-a_{0}\right)+} \\
& \left(1-\operatorname{Pr}_{t} \times \operatorname{Prob}_{t}^{\text {Transmit }}\right) \times\left(1-a_{1}\right)
\end{aligned}
$$

If ack is received:

$$
\begin{aligned}
P r_{t+\text { Tpacket }}= & \frac{\operatorname{Pr}_{t} \times \operatorname{Prob}_{t}^{\text {Transmit }} \times a_{0}}{\operatorname{Pr}_{t} \times \operatorname{Prob}_{t}^{\text {Transmit }} \times a_{0}+} \\
& \left(1-\operatorname{Pr}_{t} \times \operatorname{Prob}_{t}^{\text {Transmit }}\right) \times a_{1}
\end{aligned}
$$

If nack is received

As the assumption is that SU is aware of the beginning of PU idle time, hence we assume that PU is idle at $t=0$; Hence $P r_{0}=1$.

\section{Reward for Successful Transmission}

As the current PU idle probability is $P r_{t}$, then the probability that PU remains idle while SU is transmitting a packet (Tpacket) at time $t$ will be $P r_{t} \times \operatorname{Prob}_{t}^{\text {Transmit }}$ and the probability that SU has successfully transmitted a packet by getting an acknowledgement is:

$$
\left(1-a_{0}\right) P r_{t} \times \operatorname{Prob}_{t}^{\text {Transmit }}+\left(1-a_{1}\right)\left(1-\operatorname{Pr}_{t} \times \operatorname{Prob}_{t}^{\text {Transmit }}\right)
$$

Then the expected instantaneous benefit will be:

$B_{t}\left(P r_{t}\right)=\left[\begin{array}{l}\operatorname{Pr}_{t} \operatorname{Prob}_{t}^{\text {Transmit }}\left(1-a_{0}\right)+ \\ \left(1-P_{t} \times \operatorname{Prob}_{t}^{\text {Transmit }}\right) \\ \left(1-a_{1}\right)\end{array}\right]$ Reward $\times$ Tpacket

And the expected instantaneous collision cost will be:

$C_{t}\left(\operatorname{Pr}_{t}\right)=\left(1-\operatorname{Pr}_{t} \times \operatorname{Prob}_{t}^{\text {Transmit }}\right)$ Collcost $\times$ Tpacket 
Thus the instantaneous utility obtained by SU at time $t$ is $\quad U_{t}\left(P r_{t}\right.$, sense $)=0$ in sensing and $U_{t}\left(P r_{t}\right.$, transmit $)=B_{t}\left(P r_{t}\right)-C_{t}\left(P r_{t}\right)$ in transmitting:

$$
\begin{aligned}
& U_{t}\left(P r_{t}, \text { transmit }\right)=\left[\begin{array}{c}
\operatorname{Pr}_{t} \operatorname{Prob}_{t}^{\text {Transmit }}\left(1-a_{0}\right)+\left(1-P r_{t} \times \operatorname{Prob}_{t}^{\text {Transmit }}\right) \\
\left(1-a_{l}\right)
\end{array}\right] \\
& \text { Reward } \times \text { Tpacket }-\left(1-P_{t} \times \text { Prob }_{t}^{\text {Transmit }}\right) \\
& \text { Collcost } \times \text { Tpacket }
\end{aligned}
$$

$$
\begin{aligned}
& U_{t}\left(P_{t}, \text { transmit }\right)= \\
& {\left[\begin{array}{c}
\text { Pr }_{t} \text { Prob }_{t}^{\text {Transmit }}\left(1-a_{0}\right) \text { Reward }+ \\
\left(1-\text { Pr }_{t} \times \text { Prob }_{t}^{\text {Transmit }}\right) \\
\left\{\left(1-a_{1}\right) \text { Reward }- \text { Collcost }\right\}
\end{array}\right] \text { Tpacket }}
\end{aligned}
$$

\section{Criterion for Optimality}

The purpose is to determine a sensing transmission scheduling strategy that achieves the maximum the expected average utility per time unit.

To maximize:

$$
\lim _{L \rightarrow \infty} \frac{\left(\sum_{l=1}^{L} \sum_{t=0}^{M_{l}} U_{t}\left(\operatorname{Pr}_{t} \text { action }_{t}\right)\right) / L}{\left(\sum_{l=1}^{L}\left(I_{l}+B_{l}\right)\right) / L}
$$

Here: action $_{t}$ is SU action at time $t$ which can be sensing or transmitting, $M_{l}$ is the number of actions taken in the lth idle-busy period, $I_{l}$ is length of lth idle period and $B_{l}$ is length of lth busy period. Since $\mathrm{L}$ is very large this reduces to maximizing of $V\left(t, P r_{t}\right)=\frac{E\left[\sum_{t=0}^{M_{t}} U_{t}\left(\text { Pr }_{t}, \text { action }_{t}\right)\right]}{\bar{i}+\bar{b}}$ for an access policy of SU which defines a mapping of SU belief of PU being idle or busy to SU action of sensing or transmission. Since $\bar{i}+\bar{b}$ is fixed. The problem reduces to maximizing the numerator.

The maximum expected utility obtained by $\mathrm{SU}$ at time $t$ is then:

$$
V\left(t, P r_{t}\right)=\max _{\{\text {senses, transmit }\}}\left\{\text { Sense }\left(t, P r_{t}\right), \text { Transmit }(t, P r)\right\}
$$

Transmit $\left(t, P r_{t}\right)$ and Sense $\left(t, P r_{t}\right)$ are instantaneous expected utilities of SU by transmitting the packet and sensing the channel respectively.

From Equation (9):

$$
\begin{aligned}
& \text { Sense }\left(t, P r_{t}\right)=\sum_{i-\frac{\text { idle }}{\text { busy }}} \operatorname{Pr}\left[\text { outcome } e_{t+T \text { sense }}^{\text {sense }}=i\right] \\
& V\left(t+T \text { sense, } P r_{t+T \text { sense }}(i)\right)
\end{aligned}
$$

$$
\begin{aligned}
& \operatorname{Transmit}\left(t, P r_{t}\right)=\sum_{j=\frac{\text { ack }}{\text { nack }}} \operatorname{Pr}\left[\text { outcome }_{t+T_{\text {packet }}}^{\text {trassmit }}=j\right] \\
& V\left(t+\operatorname{Tpacket} \operatorname{Pr}_{t+\operatorname{Tpacket}}(j)\right)+U_{t}\left(\operatorname{Pr}_{t}, \text { transmit }\right)
\end{aligned}
$$

$P r_{t}$ is conditional probability of PU being idle at time $t$. The value of $P r_{t}$ at which the expected utility of transmission, Transmit $\left(t, P r_{t}\right)$ exceeds that of sensing, Sense $\left(t, P r_{t}\right)$, gives the optimal threshold: $P r_{t}{ }^{*}$.

Sense $\left(t, P r_{t}\right)$ and Transmit $\left(t, P r_{t}\right)$ intersect at $P r_{t}=$ $\mathrm{Pr}_{t}{ }^{*}$. So $\mathrm{Pr}_{t}{ }^{*}$ is that value of $\mathrm{Pr}_{t}$ at which SU should start transmission as instantaneous transmitting utility exceeds instantaneous sensing utility. Thus $P r_{t}{ }^{*}$ defines the threshold of sensing-transmission structure, when the SU should start transmission.

Lower the values of $\mathrm{Pr}_{t}{ }^{*}$ better for SU, since it can transmit even when probability of PU being idle is lower. But to protect the PU interests, cost of collision has to be introduced and increased from unity.

\section{Simulation Results}

Results for five cases of collision cost (3 to 7) have been derived. Collision cost is high in cases where PU carries critical data, which cannot afford collisions. The PU idle time is uniformly distributed in $[100,1000]$. SU transmission packet length is kept constant at 30 . Probability of detection and Probability of false alarm are 0.99 and 0.01 respectively. Reward is taken as 1 . Probability of nack due to collision with PU is taken as 0.9 and due to any other reason is then 0.1 . Thus both imperfect sensing and feedback scenarios are taken into consideration. For each case of collision cost, the SU sensing time is varied from 7 to 11 in steps of 1 .

\section{Collision Cost is 3}

It is evident from the graph in Fig. 2 and 3 that the threshold value increases with decrease in SU sensing time. It can be seen Table 1 that at the instant of time index of 100 the values of threshold for SU sensing times of $11,10,9,8$ and 7 are $0.9204,0.9272,0.9336,0.9402$ and 0.9469 . There is an increase of almost $3 \%$ in the threshold value as sensing time is decreased from 11 to 7 .

\section{Collision Cost is 4}

In this case also, the threshold value increases with decrease in SU sensing time as seen in Fig. 4. At the instant when time index is 100 the values of threshold for SU sensing times $11,10,9,8$ and 7 are 0.9421 , $0.9472,0.9519,0.9567$ and 0.962 from Table 2. There is an increase of over $2 \%$ in the threshold value as sensing time is decreased from 11 to 7 . The increase is less than in case cost of collision is 3 . The threshold is 
also higher at every time instant for the same length of sensing time in case cost of collision is increased.

\section{Collision Cost is 5, 6 and 7}

The threshold value continues to increase with decrease in SU sensing time and further increase in cost of collision as is shown in Fig. 5 to 8 . At the instant of time index 100, the value of threshold for costs of collision 5, 6 and 7 ( $\mathrm{SU}$ sensing time is 7) is 0.9711 , 0.977 and 0.9815 from Table 3 to 5 . There is an increase of $3.6 \%$ in the threshold value as cost of collision is decreased from 7 to 3 (SU sensing time is 7). The increase is $5.6 \%$ when length of SU sensing time is 11 .

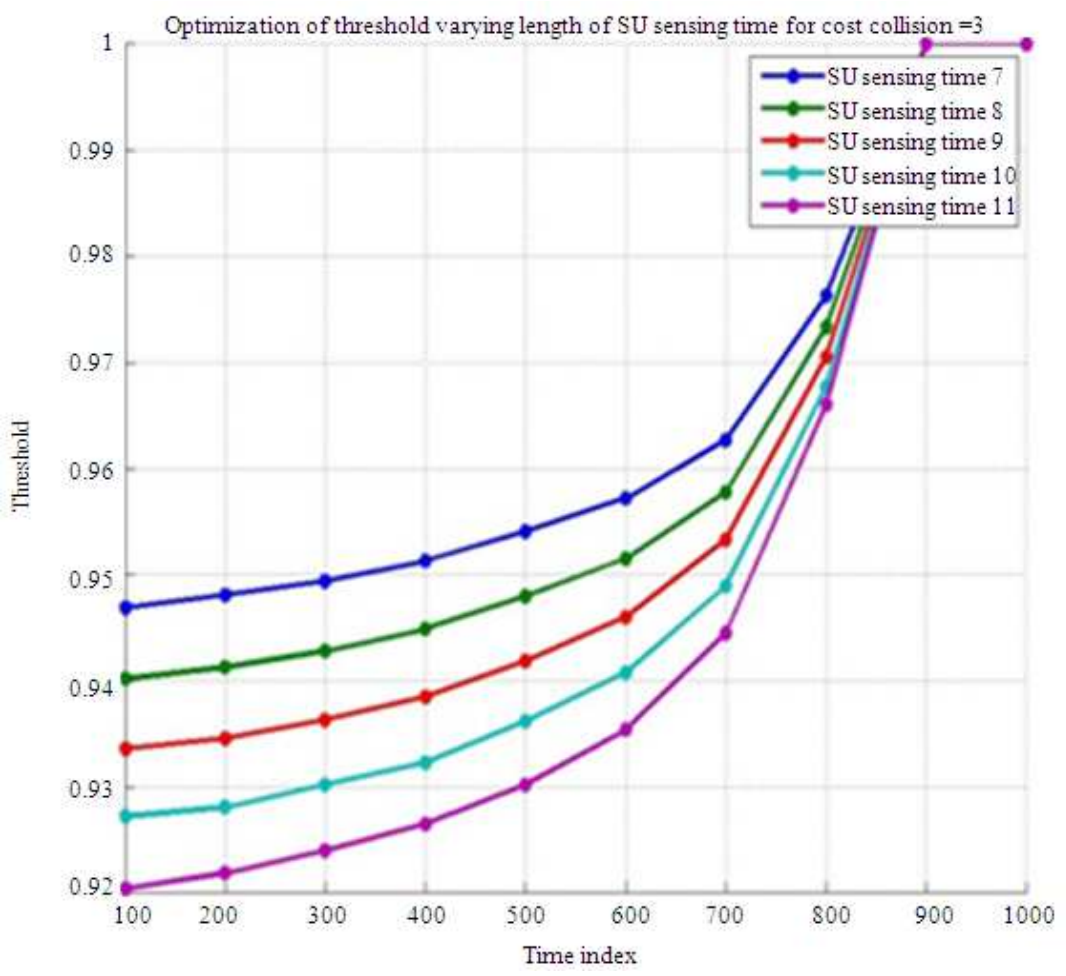

Fig. 2 . Threshold V/s time index $(\mathrm{SU}$ sensing time $=7$ to11 $)($ Collcost $=3)$

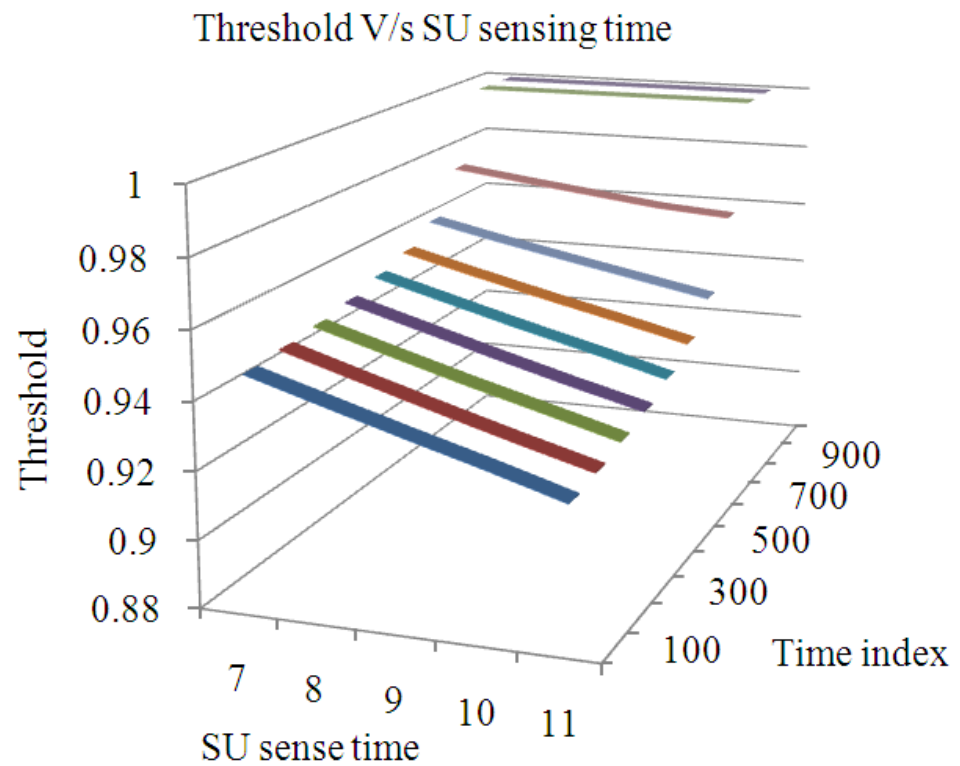

Fig. 3. Threshold V/s SU sensing time (Time index $=100-1000)($ Collcost $=3$ ) 
Inderdeep Kaur Aulakh and Renu Vig / Journal of Computer Science 2015, 11 (8): 880.891

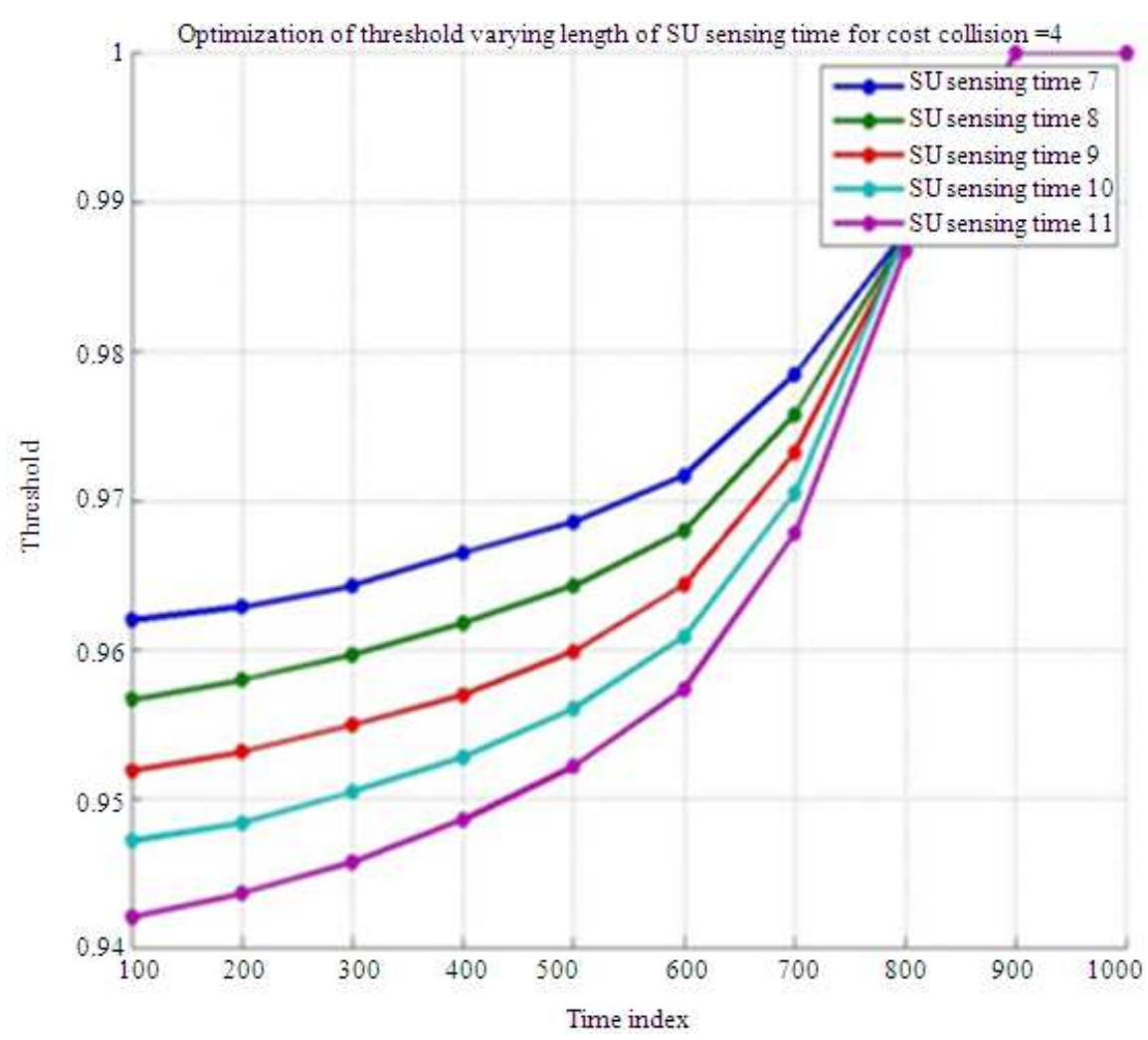

Fig. 4. Threshold V/s time index $(\mathrm{SU}$ sensing time $=7$ to11 $)($ Collcost $=4)$

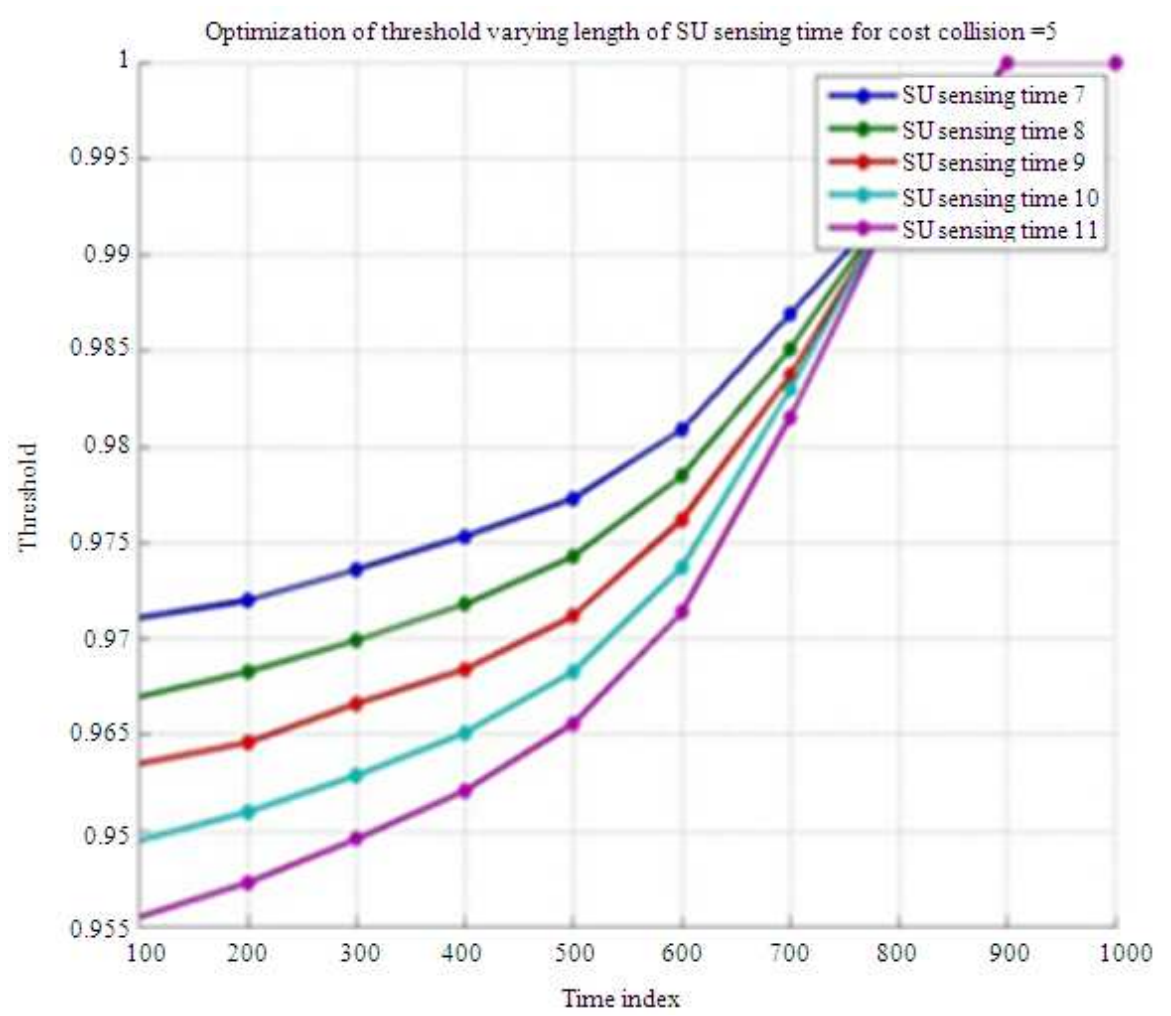

Fig. 5. Threshold V/s time index (SU sensing time $=7$ to11 $)($ Collcost $=5)$ 


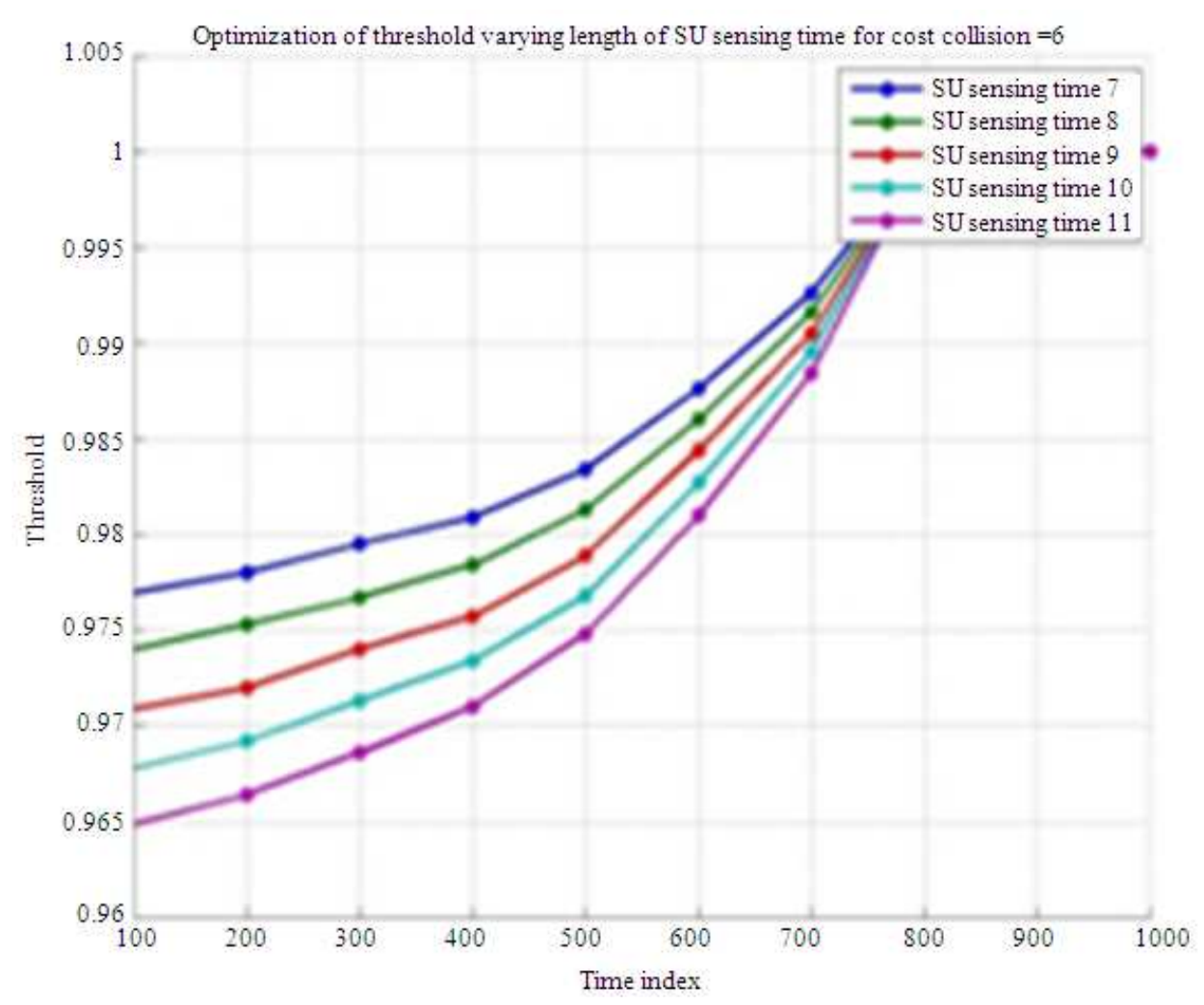

Fig. 6. Threshold V/s time index $(\mathrm{SU}$ sensing time $=7$ to11 $)($ Collcost $=6)$

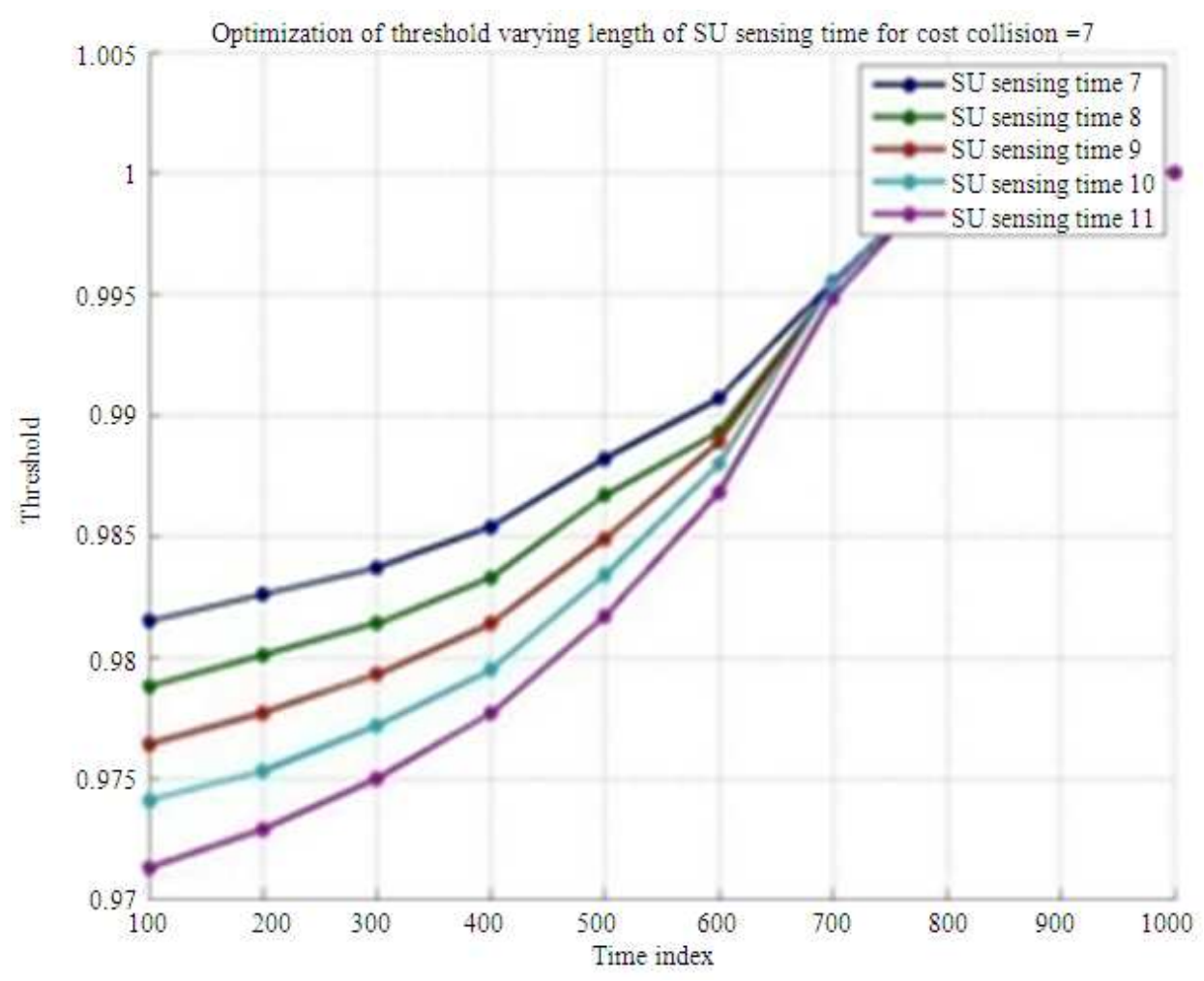

Fig. 7. Threshold V/s time index (SU sensing time $=7$ to11 $)($ Collcost $=7)$ 


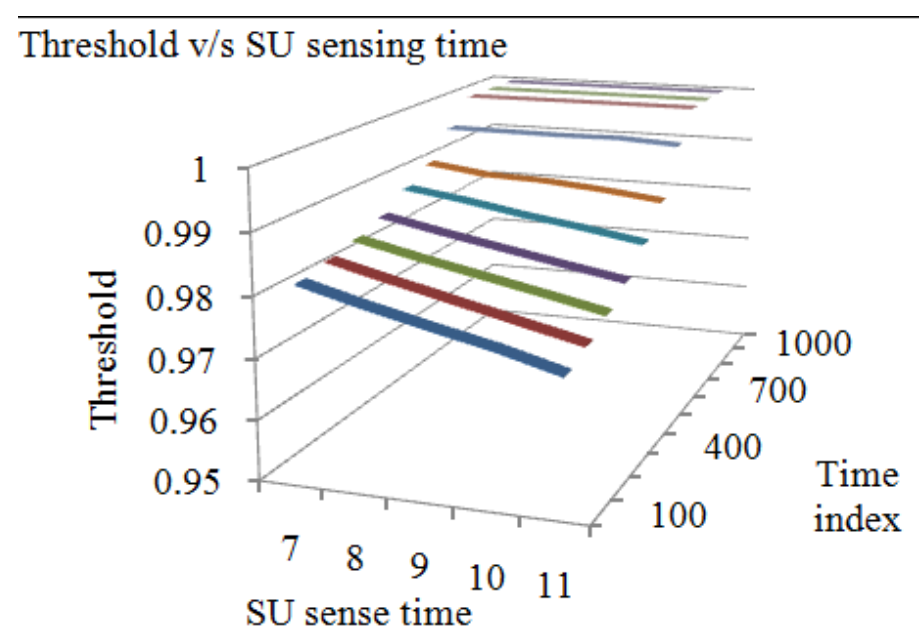

Fig. 8. Threshold V/s SU sensing time (time index $=100-1000)($ Collcost $=7$ )

Table 1. Threshold V/s time (SU sensing time $=7$ to 11$)($ Collcost $=3)$

\begin{tabular}{|c|c|c|c|c|c|}
\hline \multirow[b]{2}{*}{ Time index } & \multicolumn{5}{|c|}{ Threshold values for varying lengths of SU sensing times for Collcost $=3$} \\
\hline & Tsense $=7$ & 8 & 9 & 10 & 11 \\
\hline$\overline{100}$ & 0.9469 & 0.9402 & 0.9336 & 0.9272 & 0.9204 \\
\hline 200 & 0.9481 & 0.9413 & 0.9346 & 0.9281 & 0.9219 \\
\hline 300 & 0.9494 & 0.9428 & 0.9363 & 0.9302 & 0.9240 \\
\hline 400 & 0.9513 & 0.9449 & 0.9385 & 0.9323 & 0.9265 \\
\hline 500 & 0.9541 & 0.9480 & 0.9419 & 0.9362 & 0.9302 \\
\hline 600 & 0.9572 & 0.9515 & 0.9460 & 0.9408 & 0.9354 \\
\hline 700 & 0.9627 & 0.9578 & 0.9533 & 0.9489 & 0.9445 \\
\hline 800 & 0.9763 & 0.9733 & 0.9705 & 0.9677 & 0.9660 \\
\hline 900 & 1.0000 & 1.0000 & 1.0000 & 1.0000 & 1.0000 \\
\hline 1000 & 1.0000 & 1.0000 & 1.0000 & 1.0000 & 1.0000 \\
\hline
\end{tabular}

Table 2 . Threshold V/s time (SU sensing time $=7$ to 11$)($ Collcost $=4)$

\begin{tabular}{|c|c|c|c|c|c|}
\hline \multirow[b]{2}{*}{ Time index } & \multicolumn{5}{|c|}{ Threshold values for varying lengths of SU sensing times for Collcost $=4$} \\
\hline & Tsense $=7$ & 8 & 9 & 10 & 11 \\
\hline$\overline{100}$ & 0.962 & 0.9567 & 0.9519 & 0.9472 & 0.9421 \\
\hline 200 & 0.9629 & 0.9580 & 0.9532 & 0.9484 & 0.9437 \\
\hline 300 & 0.9643 & 0.9597 & 0.9550 & 0.9505 & 0.9458 \\
\hline 400 & 0.9665 & 0.9618 & 0.9570 & 0.9528 & 0.9486 \\
\hline 500 & 0.9686 & 0.9643 & 0.9599 & 0.9561 & 0.9522 \\
\hline 600 & 0.9717 & 0.9680 & 0.9644 & 0.9609 & 0.9574 \\
\hline 700 & 0.9785 & 0.9758 & 0.9732 & 0.9705 & 0.9678 \\
\hline 800 & 0.9880 & 0.9878 & 0.9875 & 0.9879 & 0.9867 \\
\hline 900 & 1.0000 & 1.0000 & 1.0000 & 1.0000 & 1.0000 \\
\hline 1000 & 1.0000 & 1.0000 & 1.0000 & 1.0000 & 1.0000 \\
\hline
\end{tabular}

\section{Inferences and Explanation}

Firstly optimal threshold increases with decrease in SU sensing time. This implies that SU senses more often when SU sensing time is less. Higher threshold also implies that SU throughput drops as its sensing utility is more than transmitting utility. Thus SU needs higher probability of PU being idle to start transmission. The $\mathrm{Pr}_{t}{ }^{*}$ increase with decrease in Tsense is more at Collcost $=3$ than at Collcost $=7$, hence at lower collision cost SU sensing time has more impact on optimal threshold than at higher collision cost.

Secondly optimal threshold increases with increase in cost of collision. Thus depending upon the criticality of PU data, an optimum cost of collision has to be defined. Increase in $\mathrm{Pr}_{t}{ }^{*}$ with increase in Collcost is less at Tsense $=7$ and more at $T$ sense $=11$, hence cost of collision has higher impact on optimal threshold at higher SU sensing times than at lower SU sensing times. 
Inderdeep Kaur Aulakh and Renu Vig / Journal of Computer Science 2015, 11 (8): 880.891 DOI: $10.3844 /$ jessp.2015.880.891

Table 3. Threshold V/s time (SU sensing time $=7$ to 11$)($ Collcost $=5)$ Threshold values for varying lengths of SU sensing times for Collcost $=5$

\begin{tabular}{llllll} 
& & & & & \\
Time index & Tsense $=7$ & 8 & 9 & 0.9595 & 11 \\
\hline 100 & 0.9711 & 0.9670 & 0.9635 & 0.9610 & 0.9555 \\
200 & 0.9720 & 0.9683 & 0.9646 & 0.9629 & 0.9573 \\
300 & 0.9736 & 0.9699 & 0.9666 & 0.9651 & 0.9596 \\
400 & 0.9753 & 0.9718 & 0.9684 & 0.9683 & 0.9621 \\
500 & 0.9773 & 0.9743 & 0.9712 & 0.9737 & 0.9656 \\
600 & 0.9809 & 0.9785 & 0.9762 & 0.9830 & 0.9714 \\
700 & 0.9869 & 0.9851 & 0.9837 & 0.9931 & 0.9815 \\
800 & 0.9931 & 0.9931 & 0.9931 & 1.0000 & 0.9931 \\
900 & 1.0000 & 1.0000 & 1.0000 & 1.0000 & 1.0000 \\
1000 & 1.0000 & 1.0000 & 1.0000 & & 1.0000 \\
\hline
\end{tabular}

Table 4 . Threshold V/s time (SU sensing time $=7$ to 11$)($ Collcost $=6)$

\begin{tabular}{|c|c|c|c|c|c|}
\hline \multirow[b]{2}{*}{ Time index } & \multicolumn{5}{|c|}{ Threshold values for varying lengths of SU sensing times for Collcost $=6$} \\
\hline & Tsense $=7$ & 8 & 9 & 10 & 11 \\
\hline$\overline{100}$ & 0.9770 & 0.9740 & 0.9709 & 0.9678 & 0.9649 \\
\hline 200 & 0.9780 & 0.9753 & 0.9720 & 0.9692 & 0.9664 \\
\hline 300 & 0.9795 & 0.9767 & 0.9740 & 0.9713 & 0.9686 \\
\hline 400 & 0.9809 & 0.9784 & 0.9757 & 0.9734 & 0.9710 \\
\hline 500 & 0.9834 & 0.9813 & 0.9789 & 0.9768 & 0.9748 \\
\hline 600 & 0.9876 & 0.9860 & 0.9844 & 0.9827 & 0.9810 \\
\hline 700 & 0.9926 & 0.9916 & 0.9905 & 0.9895 & 0.9884 \\
\hline 800 & 1.0000 & 1.0000 & 1.0000 & 1.0000 & 1.0000 \\
\hline 900 & 1.0000 & 1.0000 & 1.0000 & 1.0000 & 1.0000 \\
\hline 1000 & 1.0000 & 1.0000 & 1.0000 & 1.0000 & 1.0000 \\
\hline
\end{tabular}

Table 5 . Threshold V/s time ( $\mathrm{SU}$ sensing time $=7$ to 11$)($ Collcost $=7)$

\begin{tabular}{|c|c|c|c|c|c|}
\hline \multirow[b]{2}{*}{ Time index } & \multicolumn{5}{|c|}{ Threshold values for varying lengths of SU sensing times for Collcost $=7$} \\
\hline & Tsense $=7$ & 8 & 9 & 10 & 11 \\
\hline$\overline{100}$ & 0.9815 & 0.9788 & 0.9764 & 0.9741 & 0.9713 \\
\hline 200 & 0.9826 & 0.9801 & 0.9777 & 0.9753 & 0.9729 \\
\hline 300 & 0.9837 & 0.9814 & 0.9793 & 0.9772 & 0.9750 \\
\hline 400 & 0.9854 & 0.9833 & 0.9814 & 0.9795 & 0.9777 \\
\hline 500 & 0.9882 & 0.9867 & 0.9849 & 0.9834 & 0.9817 \\
\hline 600 & 0.9907 & 0.9893 & 0.9889 & 0.9880 & 0.9868 \\
\hline 700 & 0.9955 & 0.9954 & 0.9953 & 0.9954 & 0.9948 \\
\hline 800 & 1.0000 & 1.0000 & 1.0000 & 1.0000 & 1.0000 \\
\hline 900 & 1.0000 & 1.0000 & 1.0000 & 1.0000 & 1.0000 \\
\hline 1000 & 1.0000 & 1.0000 & 1.0000 & 1.0000 & 1.0000 \\
\hline
\end{tabular}

\section{Conclusion and Future Scope}

The present work has lead to traces of optimal threshold $\left(\operatorname{Pr}_{t}{ }^{*}\right)$ value against variations in length of SU sensing time (Tsense). These traces lead to determination of the optimal balance between SU aggressiveness and PU contention. With this fine tuning it will be possible to maximize the average utility. This will go on to increase the spectrum utilization efficiency. The results would lead to better and more available spectrum. They will provide an insight for the design of sensing-transmission control in cognitive networks.

This work can be extended to study the effect on optimal threshold $\left(\mathrm{Pr}_{t}{ }^{*}\right)$ for variations in parameters like probability of detection (Probd) i.e., recognizing busy PU as busy, average idle time of PU, average busy time of PU and length of SU packet (Tpacket).

\section{Funding Information}

The authors have no support or funding to report.

\section{Author's Contributions}

Inderdeep Kaur Aulakh: After undertaking an extensive study on the topic of sensing-transmission scheduling in cognitive radio networks she developed the system model, based on the constraints. Then simulated it 
in Matlab and compared the results with earlier works and also analyzed them for new implications.

Renu Vig: Designed the research plan, organized and supervised the study.

\section{Ethics}

This article is original and contains unpublished material. The corresponding author confirms that all of the other authors have read and approved the manuscript and no ethical issues involved.

\section{References}

Aulakh, I.K. and R. Vig, 2014a. Optimization of secondary user access in cognitive radio networks. Proceedings of the Recent Advances in Engineering and Computational Sciences, Mar. 6-8, IEEE Xplore Press, Chandigarh, pp: 1-6.

DOI: 10.1109/RAECS.2014.6799511

Aulakh, I.K. and R. Vig, 2014b. Optimization of SU's probability of false alarm for dynamic spectrum access in cognitive radio. Proceedings of the International Conference on Computing for Sustainable Global Development, Mar. 5-7, IEEE Xplore Press, New Delhi, pp: 710-715.

DOI: 10.1109/IndiaCom.2014.6828053

Azzalini, A., 1981. A note on the estimation of a distribution function and quantiles by a kernel method. Biometrika, 68: 326-328.

DOI: $10.1093 /$ biomet/68.1.326

Berlemann, L. and S. Mangold, 2009. Cognitive Radio and Dynamic Spectrum Access. 1st Edn., John Wiley and Sons Ltd., Chichester, ISBN-10: 0470754435, pp: 242.

Chang, N.B. and M. Liu, 2007. Optimal channel probing and transmission scheduling for opportunistic spectrum access. Proceedings of the 13th Annual ACM International Conference on Mobile Computing and Networking, Sept. 09-14, ACM, Canada, pp: 27-38. DOI: 10.1145/1287853.1287858

Chen, H.S., W. Gao and D. Daut, 2008. Spectrum sensing for wireless microphone signals. Proceedings of the 5th IEEE Annual Communications Society Conference on Sensor, Mesh and Ad Hoc Communications and Networks Workshops, Jun. 16-20, IEEE Xplore Press, San Francisco, CA., pp: 1-5. DOI: 10.1109/SAHCNW.2008.7

Chen, Y., Q. Zhao and A. Swami, 2008. Joint design and separation principle for opportunistic spectrum access in the presence of sensing errors. IEEE Trans. Inform. Theory, 54: 2053-2071. DOI: 10.1109/TIT.2008.920248
Cordeiro, C., K. Challapali, D. Birru and S.N. Shankar, 2006. IEEE 802.22: An introduction to the first wireless standard based on cognitive radios. J. Commun., 1: 38-47. DOI: 10.4304/jcm.1.1.38-47

Fette, B.A., 2009. Cognitive Radio Technology. 2nd Edn., Academic Press, Burlington, ISBN-10: 008092316X, pp: 848.

Geirhofer, S., L. Tong and B.M. Sadler, 2006. Dynamic spectrum access in WLAN channels: Empirical model and its stochastic analysis. Proceedings of the 1st International Workshop on Technology and Policy for Accessing Spectrum, (PAS'06), ACM. DOI: $10.1145 / 1234388.1234402$

Huang, S., X. Liu and Z. Ding, 2008. Short paper: On optimal sensing and transmission strategies for dynamic spectrum access. Proceedings of the 3rd IEEE Symposium on New Frontiers in Dynamic Spectrum Access Networks, Oct. 14-17, IEEE Xplore Press, Chicago, IL., pp: 1-5. DOI: 10.1109/DYSPAN.2008.91

Huang, S., X. Liu and Z. Ding, 2009a. Optimal sensingtransmission structure for dynamic spectrum access. Proceedings of the IEEE INFOCOM, Apr. 19-25, IEEE Xplore Press, Rio de Janeiro, pp: 2295-2303. DOI: 10.1109/INFCOM.2009.5062155

Huang, S., X. Liu and Z. Ding, 2009b. Optimal transmission strategies for dynamic spectrum access in cognitive radio networks. IEEE Trans. Mobile Comput., 8: 1636-1648. DOI: 10.1109/TMC.2009.84

Kim, H. and K.G. Shin, 2008. Efficient discovery of spectrum opportunities with MAC-layer sensing in cognitive radio networks. IEEE Trans. Mobile Comput., 7: 533-545. DOI: 10.1109/TMC.2007.70751

Lai, L., H.E. Gamal, H. Jiang and H.V. Poor, 2007. Cognitive medium access: Exploration, exploitation and competition.

Liang, Y.C., Y. Zeng, E. Peh and A.T. Hoang, 2008. Sensing-throughput tradeoff for cognitive radio networks. IEEE Trans. Wireless Commun., 7: 13261337. DOI: 10.1109/TWC.2008.060869

Liu, Q., X. Wang and Y. Cui, 2012. Adaptive Scheduling Of Sequential Periodic Sensing For Cognitive Radios. Proceedings of the IEEE INFOCOM, (COM'12), IEEE Xplore Press, Turin, Italy.

Mokhtar, R.A., S. Khatun, B.M. Ali and A. Ramli, 2009. Cooperative sensing in cognitive radio networks-avoid non-perfect reporting channel. Am. J. Eng. Applied Sci., 2: 471-475. DOI: 10.3844 /ajeassp.2009.471.475

Wang, P., L. Xiao, S. Zhou and J. Wang, 2007. Optimization of detection time for channel efficiency in cognitive radio systems. Proceedings of the IEEE Wireless Communications and Networking Conference, Mar. 11-15, IEEE Xplore Press, Kowloon, pp: 111-115. DOI: $10.1109 /$ WCNC.2007.26 
Willkomm, D., S. Machiraju, J. Bolot and A. Wolisz, 2008. Primary users in cellular networks: A largescale measurement study. Proceedings of the 3rd IEEE Symposium on New Frontiers in Dynamic Spectrum Access Networks, Oct. 14-17, IEEE Xplore Press, Chicago IL., pp: 1-11.

DOI: 10.1109/DYSPAN.2008.48

Zhang, T. and D.H.K. Tsang, 2011. Optimal cooperative sensing scheduling for energy-efficient cognitive radio networks. Proceedings of the IEEE INFOCOM, Apr. 10-15, IEEE Xplore Press, Shanghai, pp: 27232731. DOI: 10.1109/INFCOM.2011.5935104
Zhao, Q. and K. Liu, 2008. Detecting, tracking and exploiting spectrum opportunities in unslotted primary systems. Proceedings of the IEEE Radio and Wireless Symposium, Jan. 22-24, IEEE Xplore Press, Orlando, FL., pp: 491-494. DOI: $10.1109 /$ RWS.2008.4463536

Zhao, Q., S. Geirhofer, L. Tong and B.M. Sadler, 2007. Optimal dynamic spectrum access via periodic channel sensing. Proceedings of the IEEE Wireless Communications and Networking Conference, Mar. 11-15, IEEE Xplore Press, Kowloon, pp: 33-37. DOI: 10.1109/WCNC.2007.12 